\title{
Generalizing the Petr-Douglas-Neumann Theorem on $n$-gons
}

\author{
Stephen B. Gray
}

1. INTRODUCTION: THE PDN-THEOREM. A popular topic in plane Euclidean geometry is the construction of similar figures on each side of a polygon, and the properties of the new polygon so formed. This subject, still active, has been investigated for over a century. Merriell [18] surveys the field, mentioning constructions using similar triangles as far back as 1870 . Probably the best known such theorem is "Napoleon's theorem," dating to 1825 or earlier, in which the polygons as well as the constructed figures are triangles. This famous result has had innumerable generalizations, some of which are discussed and carried further here.

One of the most surprising such extensions involves successive levels or "stages" of constructions on $n$-gons. One concise statement of this theorem is Neumann's [19], which, somewhat modified, states: If isosceles triangles with apex angles $2 k \pi / n$ are erected on the sides of an arbitrary $n$-gon $\boldsymbol{A}_{0}$, and if this process is repeated with the $n$-gon formed by the free apices of the triangles, but with a different value of $k$, and so on until all values $1 \leq k \leq n-2$ have been used (in arbitrary order), then a regular $n$-gon $\boldsymbol{A}_{n-2}$ is formed whose centroid coincides with the centroid of $\boldsymbol{A}_{0}$.

Centroid invariance is the origin of the phrase "concentric polygons" [18], sometimes used to describe this area of geometry. This field is part of what can be called linear geometry, because all constructed points are linear functions of the vertices of the initial polygon. Of course, linearity encourages proofs using matrices and other linear algebra methods.

The only flexibility in this theorem is choosing the order in which the values of $k$ are used. There are $(n-1)$ ! such permutations, all leading to the same final point, the centroid of $\boldsymbol{A}_{0}$. This stands in contrast to the new theorem, introduced in Section 2, that has $n-1$ free geometric variables.

Karel Petr of Prague was the first to publish this theorem, which he did in 1908 [23]. His name is sometimes associated with it [21], but his paper received less attention than two others, by Douglas in 1940 [8, p. 112] and Neumann in 1941 [19, p. 236]. They each independently rediscovered it, as I also did in 1961. Their papers were followed in the next two years by several more: in [9], Douglas introduces cyclic (circulant) matrices [6], showing that their inherent commutation implies that the construction stages commute, which can readily be proved geometrically. In [1], Baker provides another proof, avoiding Neumann's symmetrical components. In the same issue of the same journal, Neumann published a further simplification [20]. An article by Fisher, Ruoff, and Shiletto [12, p. 329] contains a short algebraic proof, whose background is also developed in the same article. In two papers [4], [5], Chang provides two proofs using circulant matrices. All these proofs use complex numbers and roots of unity, as does mine. Bogomolny [3] provides an interactive facility for dynamically exploring this theorem, and gives a concise proof. In related work, Wong [25] discusses polygons, inverses, and circulant matrices in general.

I will call this result the PDN-theorem, for Petr, Douglas, and Neumann, in an attempt to eliminate considerable name confusion. This theorem has been called by at least four names: "Douglas's theorem" [16], the "Douglas-Neumann theorem" [26], 
the "Napoleon-Douglas-Neumann theorem" [5], and "Petr's theorem" [21]. To add to the confusion, there are at least two other much simpler results often called the "Douglas-Neumann theorem" (for one, see [12, p. 330]). There are also at least two unrelated results called "Douglas's theorem" (one is referred to in [17]). Using the suggested new name would reduce ambiguity, preserve recognizability, and properly credit Petr, whose contribution is usually overlooked.

The PDN-theorem is one of the few Euclidean propositions that have a discrete variable $n$ in their definitions. Several such theorems are given in [18, thm. 8] and in [15] that emphasize ordinary induction on integers having geometric meaning. The PDNtheorem has never been proved using induction, perhaps because as it proceeds from $n$-gons to $(n+1)$-gons, all the triangles used change discontinuously. As $n$ increases, the number of constructed triangles goes up as $\mathrm{O}\left(n^{2}\right)$, so for even moderate values of $n$ (greater than ten, say) a full illustration of the theorem is almost impossible to understand visually. It is not surprising that no synthetic proof has ever been presented, nor will one be given here.

All proof methods used to date for the PDN-theorem strictly depend on the similarity of all triangles used in any one stage of the construction. This restriction appears to be intrinsic to the problem, which is perhaps why the theorem has never been generalized beyond the similarity constraint. This may seem surprising, in that the theorem is almost one hundred years old and still receives attention [21]. In fact it has been called "remarkable" [16, p. 38] and "beautiful" [26, p. 471] and certainly possesses inherent interest. But I have found only one previous generalization, that of Wong [26]. He presents conditions that make the final $n$-gon a proper $(n / m)$-gon traced $m$ times, and gives related results. His development, like most of the others, uses complex variables but no matrix theory.

2. THE NEW RESULT. The present paper takes a different point of view, generalizing the PDN-theorem while using a proof method that is more elementary (but not necessarily simpler) than existing proofs. The new result also involves $n-1$ stages of triangle construction that finally converge to a single point. However here the triangles are not specified numerically by angle. Instead, they originate from the combination of an arbitrary "model $n$-gon" $\boldsymbol{Z}$ and a separate common vertex point $Y$, as shown on the left in Figures 1 and 2. There are a total of $n(n-1) / 2$ distinct model triangles, each used twice, with $n$ of them used at each stage of the construction. In general, none of the model triangles is similar to any of the others. This is why previous techniques used for the PDN-theorem, such as roots of unity [8, p. 108], symmetric components [19], and circulant matrices [6], do not apply here.

As might be expected, certain properties of PDN-theorem constructions do not carry over to the present generalization. First, centroids are not preserved in general, so this theorem does not create concentric figures. Second, the stages of construction do not commute. Third and most important, the $n$-gon resulting from the last of the $n-2$ stages is not regular, but instead is geometrically similar to the model polygon $\boldsymbol{Z}$. In a doubly specialized case, $Z$ is a regular $n$-gon and $Y$ is its centroid, so that the model triangles used in any one stage are similar and isosceles, and the final polygon is regular. This is exactly the PDN-theorem.

The nature of the present generalization derives largely from defining the construction objects in a "model," shown on the left in Figures 1 and 2. (Figure 2 shows the same model twice, with different connecting lines. The model figure is separate from the theorem itself, seen on the right in the figures. This separation is necessary for both visual and conceptual clarity. The model has the property that the separate common vertex $Y$ can be located anywhere in the plane of $\boldsymbol{Z}$. (N.B. If $Y$ lies on a side of $\boldsymbol{Z}$, one 
of the model's constituent triangles degenerates to a line segment; if $Y$ coincides with a vertex of $\boldsymbol{Z}$, two of the model's triangles degenerate to different line segments. In both cases the theorem holds.) The model figure has two restrictions. First, no vertices of the $n$-gon $\boldsymbol{Z}$ itself may coincide. Second, the model triangles must fit together to form an $n$-gon, having no gaps or steps; see Figure 1 (left) and Figure 2 (left, top). It can be shown either algebraically or geometrically that this criterion is necessary and sufficient to ensure that the constructions are "noninvertible," defined as follows.

Suppose $\boldsymbol{T}$ is a specific one-stage triangle construction that can be applied to an arbitrary $n$-gon $\boldsymbol{A}$ to yield a derived $n$-gon $\boldsymbol{B}$. Then we can write $\boldsymbol{B}=\boldsymbol{T A}$. If $\boldsymbol{T}$ is invertible, by definition its inverse is constructible, so that for an arbitrary $\boldsymbol{B}$ we can always construct $\boldsymbol{A}=\boldsymbol{T}^{-1} \boldsymbol{B}$. Invertible constructions are of no interest here, because they cannot create the specialized $n$-gons that are the chief subject of this paper.

The proofs of the present theorem given in Sections 5, 6, and 7 implicitly demonstrate that the constructions are noninvertible, so we do not need to prove this separately.

3. SPECIAL CASE: $\boldsymbol{n}=3$. Figure 1 (left) shows the model 3-gon $\boldsymbol{Z}$ and its constituent triangles. The 3 -gon's vertices are labelled $Z_{0}, Z_{1}$, and $Z_{2}$. The common vertex $Y$, placed inside $Z$ for visual clarity, is part of the three model triangles $Y Z_{0} Z_{1}$, $Y Z_{1} Z_{2}$, and $Y Z_{2} Z_{0}$. Figure 1 (right) illustrates the theorem itself, which starts with an arbitrary original 3-gon $\boldsymbol{A}_{0}=A_{00} A_{01} A_{02}$, in general not similar to $\boldsymbol{Z}$. Triangle $\boldsymbol{A}_{0}$ is pictured with a dashed line. In a first stage of construction $(t=1, t$ denoting the current stage), triangles directly similar (similar without mirroring) to the model triangles are constructed on the sides of $\boldsymbol{A}_{0}$, with vertices placed as follows: $Z_{1}$ and $Z_{0}$ are mounted on $A_{00}$ and $A_{01}$, respectively; $Z_{2}$ and $Z_{1}$ are put on $A_{01}$ and $A_{02}$; and $Z_{0}$ and $Z_{2}$ are on $A_{02}$ and $A_{00}$. The three new vertices, each corresponding to the model vertex $Y$, form a 3-gon $\boldsymbol{A}_{1}=A_{10} A_{11} A_{12}$, which is shown in thick lines. The construction triangles themselves are represented by thin solid lines. The result is that $\boldsymbol{A}_{1}$ is similar to $\boldsymbol{Z}$, a fact to be proved later. On the sides of $\boldsymbol{A}_{1}$ we do a second $(t=2)$ construction using the same model triangles again but in the opposite sense: $Z_{0} Z_{1}$ is put on $A_{11} A_{12}$,

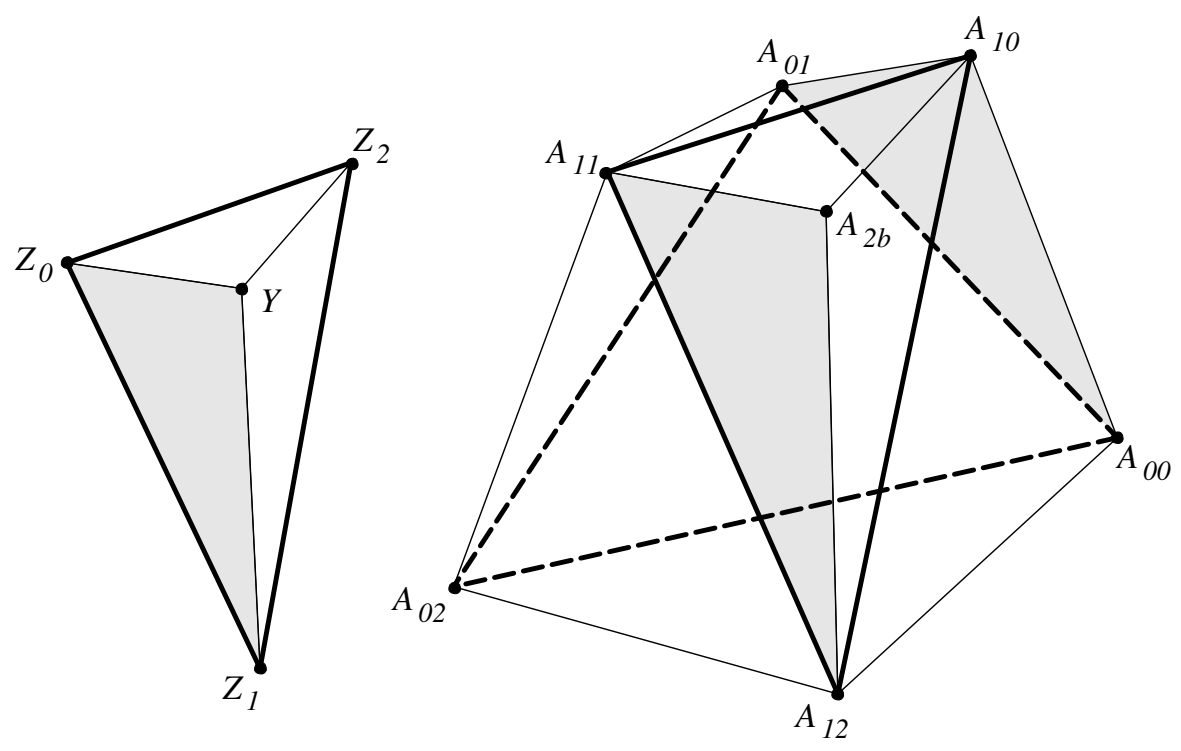

Figure 1. The case $n=3$ : model (left); theorem (right). 
$Z_{1} Z_{2}$ on $A_{12} A_{10}$, and $Z_{2} Z_{0}$ on $A_{10} A_{11}$. Assuming for the moment that $A_{1}$ is similar to $Z$, it follows that the three new vertices coincide in one point; this is given the general vertex label $A_{2 b}, b=0,1,2$, emphasizing that it is a (degenerate) triangle. This point is not related in a simple way to the centroid of $\boldsymbol{A}_{0}$, even if $Y$ is the centroid of $\boldsymbol{Z}$. To clarify Figure 1 further, one model triangle and the two triangles similar to it in the theorem construction are shaded.

The theorem, to be stated formally in Section 7, is true whether $Y$ is inside, outside, or on the triangle $\boldsymbol{Z}$. It also holds if any of the vertices of $\boldsymbol{A}_{0}$ is moved through its opposite side so that the three triangles constructed first lie on the inward side of $\boldsymbol{A}_{0}$; this continuity unifies the inward and outward cases.

The above generalization for $n=3$ is not new, being shown in Figure 12 of [13] and in Theorem 3.1 of Rigby in slightly different forms. However it has quite a few immediate implications that are not usually associated with it; some of these follow.

1. When $\boldsymbol{Z}$ is equilateral and $Y$ is its centroid, $\boldsymbol{A}_{1}$ is equilateral. This is just Napoleon's theorem and has been proven in many different ways. In Exercise $22 \mathrm{a}$ of $[\mathbf{2 8}]$ it is proved using a series of $120^{\circ}$ rotations. (Rotations and dilations about a common center, or spiral similarities, are powerful techniques for some theorems like the present one; see [11], [28] and [29].) An excellent survey paper [27] gives many implications and converses of Napoleon's theorem.

2. In Figure 1 (left) if $Y$ is the circumcenter of $Z_{0} Z_{1} Z_{2}$, certain angle relationships hold, namely, $\angle A_{12} A_{10} A_{11}=\angle A_{00} A_{10} A_{01} / 2, \angle A_{11} A_{12} A_{10}=\angle A_{02} A_{12} A_{00} / 2$, and $\angle A_{10} A_{11} A_{12}=\angle A_{01} A_{11} A_{02} / 2$. This is Exercise 22b in [28] and follows easily from the present theorem.

3. Let $\boldsymbol{T}=A_{1} A_{2} A_{3}$ be an arbitrary triangle. Construct points $P_{1}, P_{2}$, and $P_{3}$ external to $\boldsymbol{T}$, such that $\angle P_{1} A_{2} A_{3}=\angle P_{1} A_{3} A_{2}=15^{\circ} ; \angle P_{2} A_{3} A_{1}=45^{\circ} ; \angle P_{2} A_{1} A_{3}=$ $30^{\circ} ; \angle P_{3} A_{2} A_{1}=45^{\circ} ; \angle P_{3} A_{1} A_{2}=30^{\circ}$. Then $P_{1} P_{2} P_{3}$ is a $90^{\circ}-45^{\circ}-90^{\circ}$ triangle. (Notation is from the original [2].)

4. Given a triangle $A B C$, erect equilateral triangles $B A P$ and $A C Q$ outwardly on sides $A B$ and $C A$. Let $R$ be the midpoint of side $B C$, and let $G$ be the centroid of triangle $A C Q$. Then triangle $P R G$ is $30^{\circ}-90^{\circ}-60^{\circ}$ [14].

5. If squares are constructed on the sides of a triangle towards the triangle's exterior, then the segment joining two of the centers is perpendicular and congruent to the segment joining the third center to the vertex opposite it (see [11, thm. 2]).

6. Let $B Z C$ and $C X A$ be nondegenerate triangles (with vertices corresponding in the order given) constructed both towards the exterior or both towards the interior of arbitrary triangle $A B C$. Let $\angle B Z C=\angle C X A=\beta$. Let $M$ be the point in the plane that is equidistant from $A$ and $B$ and that is located so that $\angle B M A=2 \beta$. Then $M Z=M X($ see $[\mathbf{1 1}$, thm. 5]).

7. Let the model triangle in Figure 1 be made degenerate so that all four points lie on a line. The obvious identity

$$
\left(Y Z_{0} / Y Z_{1}\right)\left(Y Z_{1} / Y Z_{2}\right)\left(Y Z_{2} / Y Z_{0}\right)=1
$$

relates the lengths of segments on this line. These lengths are proportional to corresponding segments in the theorem figure, since both are limiting cases of similar triangles. In the theorem figure the previous expression becomes the nonobvious identity

$$
\left(A_{10} A_{01} / A_{01} A_{00}\right)\left(A_{11} A_{02} / A_{11} A_{01}\right)\left(A_{12} A_{00} / A_{12} A_{02}\right)=1 .
$$


Drawing the theorem figure for this case, one can see that it is an illustration of the theorem of Menelaus, which is therefore a special case of the present proposition. The statement of this theorem on page 28 of [22] reads: "A line cuts the sides $B C, C A$, and $A B$ of a triangle $A B C$ in the points $L, M$, and $N$ respectively. In terms of signed segments, $(B L: L C)(C M: M A)(A N: N B)=-1$." (This -1 is compatible with the +1 in the previous identity because the line segments were arbitrarily assigned different directions.)

4. SPECIAL CASE: $\boldsymbol{n}=\mathbf{4}$. Here we use $n-1=3$ stages of construction to create the final point, just as in the PDN-theorem. They are all derived from the same model 4-gon $Z$, illustrated twice on the left side of Figure 2. In both pictures of the model, points $Z=Z_{0} Z_{1} Z_{2} Z_{3}$ are located identically, as is the common vertex $Y$. The model is drawn twice to clarify the use of different triangles at different stages of construction.

Both models together define six triangles with $Y$ as common vertex. These six are each used twice, for a total of $12=n(n-1)$ triangles. The $t=1$ construction stage uses the four model triangles whose second subscript minus the first equals one modulo 4 (for example, $Z_{0} Z_{1} Y$ in the top model). For the $t=2$ stage the two triangles whose subscript difference is two modulo 4 (for example, $Z_{1} Z_{3} Y$ in the bottom model) are used, twice each. In the third stage the second subscript minus the first is three modulo 4, for example $Z_{3} Z_{2} Y$, implying that the triangles from the top model are used again.

The subscript difference is called the vertex spacing parameter $q(t)$. The model triangles used in stage $t$ of the construction are $Z_{k} Z_{k+q(t)} Y$, where $0 \leq k \leq n-1$, $1 \leq q(t) \leq n-1$, and subscripts are taken modulo $n$. This relation will be further defined in Section 7. In all cases of the theorem in the present paper, $q(t)=t$ for any $n$. Although it is not strictly necessary, we distinguish between $q(t)$ and $t$ itself to emphasize their different meanings, and because I am working on variations of the present theorem in which $q(t) \neq t$.

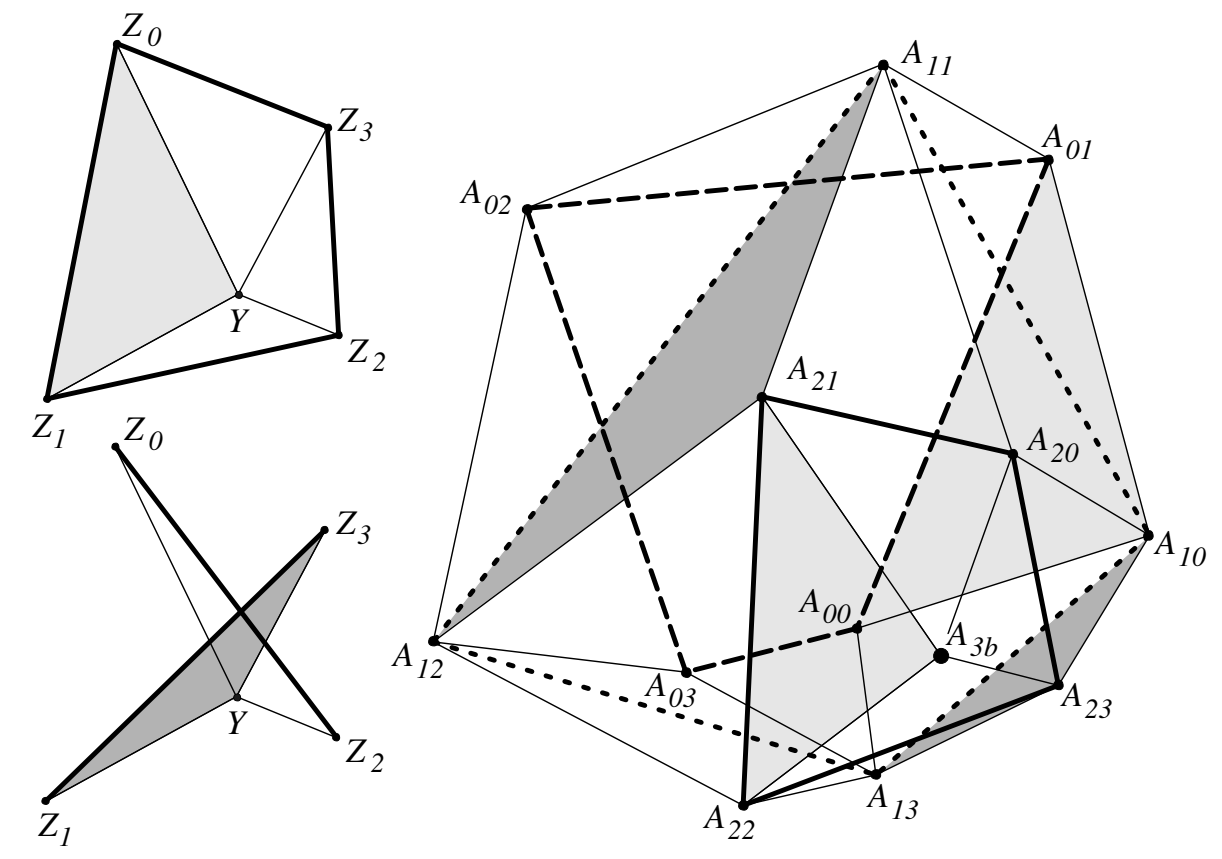

Figure 2. The case $n=4$ : models (left); theorem (right). 
In Figure 2, the shading indicates correspondence between the models and the theorem figure. In the $t=1$ stage of the construction, model triangles $Z_{0} Z_{1} Y$, $Z_{1} Z_{2} Y, Z_{2} Z_{3} Y$, and $Z_{3} Z_{0} Y(q=1)$ are used first, mounted on the sides of the original 4-gon $\boldsymbol{A}_{0}=A_{00} A_{01} A_{02} A_{03}$, shown with dashed lines. This creates a new 4-gon $A_{1}=A_{10} A_{11} A_{12} A_{13}$, displayed with dotted lines. As before, the first triangle is placed so that $Z_{0}$ is on $A_{01}$ and $Z_{1}$ is on $A_{00}$; for the second, $Z_{1}$ is on $A_{02}$ and $Z_{2}$ is on $A_{01}$, and so on. For the $t=2$ stage, triangles $Z_{2} Z_{0} Y$ and $Z_{3} Z_{1} Y(q=2)$ are mounted on $A_{1}$ in the positions indicated. Vertices $Z_{2}$ and $Z_{0}$ are placed on $A_{10}$ and $A_{11}$ and on $A_{13}$ and $A_{12}$, respectively, giving $A_{20}$ and $A_{22} ; Z_{3}$ and $Z_{1}$ are placed on $A_{10}$ and $A_{13}$ and on $A_{11}$ and $A_{12}$, respectively, producing $A_{23}$ and $A_{21}$. The resulting polygon $\boldsymbol{A}_{2}=A_{20} A_{21} A_{22} A_{23}$, drawn with thick solid lines, is similar to $\boldsymbol{Z}=Z_{3} Z_{0} Z_{1} Z_{2}$. In the final $t=3$ stage, the model triangles corresponding to $q=3$ are used, pointing inward on the sides of $\boldsymbol{A}_{2}$ and yielding a single point. This point is labelled $A_{3 b}$ to emphasize that it is a degenerate quadrilateral. An explicit rule for placing triangles on the sides of $n$-gons is defined by the general construction matrices discussed in Section 7.

For any given $n$, the model figure is fully determined by $n-1$ mutually independent triangles; that is, each of these can have arbitrary angles and side length ratios unrelated to those of the others. Figure 2 (left) shows that the rest of the $n(n-1) / 2$ model triangles are then completely defined. The present general theorem can produce many specialized results because it has $2 n-2$ independent real variables, whereas the PDNtheorem has no free variables for a given starting $n$-gon. A few resulting specializations follow.

The case when $\boldsymbol{Z}$ is a square and $Y$ is its centroid is the long-known Van Aubel theorem (see Exercise 24b in [28]). It has been generalized several times [7], but the following corollary appears to be new: in the notation of Figure 2, if $\boldsymbol{Z}$ is square, then whether $Y$ is its centroid or not, $\boldsymbol{A}_{2}$ is square and the diagonals of $\boldsymbol{A}_{1}$ are equal and perpendicular. Another apparently new generalization of Van Aubel's theorem is obtained by placing $Y$ at the intersection of the diagonals of the model. Another choice is to put $Y$ at one of the vertices $Z_{k}$; a simpler figure and theorem result. If two adjacent vertices of $\boldsymbol{A}_{0}$ coincide, several propositions about triangles appear.

5. MATRIX PROOF FOR $\boldsymbol{n}=3$. Referring to Figure 1, we wish to construct a triangle directly similar to $Y Z_{0} Z_{1}$ on side $A_{01} A_{00}$ of the theorem figure, among two other like constructions. Vertex $Z_{0}$ is to go on $A_{01}, Z_{1}$ on $A_{00}$, and the common vertex $Y$ will define the position of $A_{10}$. Treating all vertices as complex numbers, and noting that two triangles are similar if two corresponding sides have equal ratios, $A_{10}$ is determined by

$$
\frac{A_{10}-A_{00}}{A_{01}-A_{00}}=\frac{Y-Z_{1}}{Z_{0}-Z_{1}} .
$$

Next, we define for any $Z_{u}$ and $Z_{v}$, assuming $u \neq v$ and $Z_{u} \neq Z_{v}$,

$$
Z_{u v}=\frac{Z_{u}-Y}{Z_{u}-Z_{v}}
$$

Two easily established identities will be useful later for purposes of simplification:

$$
Z_{u v}+Z_{v u}=1,
$$

and

$$
Z_{w u} Z_{u v}+Z_{w v} Z_{v u}=Z_{w u} Z_{w v}
$$


Combining (1) and (2) gives

$$
A_{10}=A_{00} Z_{01}+A_{01} Z_{10}
$$

and similarly for the other vertices, always taking subscripts modulo 3 (in the general case, modulo $n$ ). (It is helpful to remember that in equations such as (5), the second subscript on $A$ and the second subscript on $Z$ indicate the vertices that coincide. In other words, $Z_{1}$ is placed on $A_{00}$ and $Z_{0}$ on $A_{01}$, as in the figures.) Generalizing from (5), all the new vertices from the $t=1$ stage of construction can be written in terms of polygon vertex vectors and construction matrices as

$$
\left[\begin{array}{c}
A_{10} \\
A_{11} \\
A_{12}
\end{array}\right]=\left[\begin{array}{ccc}
Z_{01} & Z_{10} & 0 \\
0 & Z_{12} & Z_{21} \\
Z_{02} & 0 & Z_{20}
\end{array}\right]\left[\begin{array}{c}
A_{00} \\
A_{01} \\
A_{02}
\end{array}\right],
$$

or identifying a polygon with its column vector of vertices, $\boldsymbol{A}_{1}=\boldsymbol{M}_{31} \boldsymbol{A}_{0}$, where $\boldsymbol{M}_{31}$ is the square matrix in (6). (The general notation is $\boldsymbol{M}_{n t}$.) The $t=2$ construction is seen from Figure 1 to be

$$
\left[\begin{array}{l}
A_{20} \\
A_{21} \\
A_{22}
\end{array}\right]=\left[\begin{array}{ccc}
Z_{02} & Z_{20} & 0 \\
0 & Z_{10} & Z_{01} \\
Z_{12} & 0 & Z_{21}
\end{array}\right]\left[\begin{array}{l}
A_{10} \\
A_{11} \\
A_{12}
\end{array}\right]
$$

or $\boldsymbol{A}_{2}=\boldsymbol{M}_{32} \boldsymbol{A}_{1}=\boldsymbol{M}_{32} \boldsymbol{M}_{31} \boldsymbol{A}_{0}$. The two successive stages are

$$
A_{2}=Q_{32} A_{0}
$$

where $\boldsymbol{Q}_{32}=\boldsymbol{M}_{32} \boldsymbol{M}_{31}$. (In the general case the corresponding matrix that incorporates all stages of construction is $\boldsymbol{Q}_{n, n-1}$.) Calculating $\boldsymbol{M}_{32} \boldsymbol{M}_{31}$ with the help of definition (2) and identities (3) and (4) gives

$$
\boldsymbol{Q}_{32}=\left[\begin{array}{ccc}
Z_{01} Z_{02} & Z_{12} Z_{10} & Z_{20} Z_{21} \\
Z_{01} Z_{02} & Z_{12} Z_{10} & Z_{20} Z_{21} \\
Z_{01} Z_{02} & Z_{12} Z_{10} & Z_{20} Z_{21}
\end{array}\right]
$$

All rows are identical, so the vertices of $\boldsymbol{A}_{2}$ coincide, and are given the general vertex label $A_{2 b}$. From (8) and (9) we obtain

$$
A_{2 b}=A_{20}=A_{21}=A_{22}=Z_{01} Z_{02} A_{00}+Z_{12} Z_{10} A_{01}+Z_{20} Z_{21} A_{02}
$$

It is easy to verify that the sum of each row in $\boldsymbol{M}_{31}, \boldsymbol{M}_{32}$, and $\boldsymbol{Q}_{32}$ is 1 , as required for the matrix representation to be origin-independent. It is also easy to check that matrices of the forms (6), (7), and (9) are noninvertible, meaning that the successive polygons so constructed are increasingly specialized.

6. MATRIX PROOF FOR $\boldsymbol{n}=4$. The rule for constructing each stage of triangles is further illustrated by the $n=4$ case, where we simply exhibit the three construction matrices. Analogously to the situation for $n=3$, we have $A_{3}=\boldsymbol{M}_{43} \boldsymbol{A}_{2}=$ $\boldsymbol{M}_{43} \boldsymbol{M}_{42} \boldsymbol{A}_{1}=\boldsymbol{M}_{43} \boldsymbol{M}_{42} \boldsymbol{M}_{41} \boldsymbol{A}_{0}$, where the $\boldsymbol{A}_{t}$ are now 4-by-1 column matrices, and

$$
\boldsymbol{M}_{41}=\left[\begin{array}{cccc}
Z_{01} & Z_{10} & 0 & 0 \\
0 & Z_{12} & Z_{21} & 0 \\
0 & 0 & Z_{23} & Z_{32} \\
Z_{03} & 0 & 0 & Z_{30}
\end{array}\right], \quad \boldsymbol{M}_{42}=\left[\begin{array}{cccc}
Z_{02} & Z_{20} & 0 & 0 \\
0 & Z_{13} & Z_{31} & 0 \\
0 & 0 & Z_{20} & Z_{02} \\
Z_{13} & 0 & 0 & Z_{31}
\end{array}\right]
$$




$$
\boldsymbol{M}_{43}=\left[\begin{array}{cccc}
Z_{03} & Z_{30} & 0 & 0 \\
0 & Z_{10} & Z_{01} & 0 \\
0 & 0 & Z_{21} & Z_{12} \\
Z_{23} & 0 & 0 & Z_{32}
\end{array}\right]
$$

The relevant products of the matrices in (11) are found to be $\boldsymbol{Q}_{41}=\boldsymbol{M}_{41}$,

$$
\boldsymbol{Q}_{42}=\boldsymbol{M}_{42} \boldsymbol{M}_{41}=\left[\begin{array}{cccc}
Z_{01} Z_{02} & Z_{10} Z_{12} & Z_{20} Z_{21} & 0 \\
0 & Z_{12} Z_{13} & Z_{21} Z_{23} & Z_{31} Z_{32} \\
Z_{02} Z_{03} & 0 & Z_{23} Z_{20} & Z_{32} Z_{30} \\
Z_{03} Z_{01} & Z_{13} Z_{10} & 0 & Z_{30} Z_{31}
\end{array}\right]
$$

and

$$
\boldsymbol{Q}_{43}=\boldsymbol{M}_{43} \boldsymbol{M}_{42} \boldsymbol{M}_{41}=\left[\begin{array}{cccc}
Z_{01} Z_{02} Z_{03} & Z_{12} Z_{13} Z_{10} & Z_{23} Z_{20} Z_{21} & Z_{30} Z_{31} Z_{32} \\
Z_{01} Z_{02} Z_{03} & Z_{12} Z_{13} Z_{10} & Z_{23} Z_{20} Z_{21} & Z_{30} Z_{31} Z_{32} \\
Z_{01} Z_{02} Z_{03} & Z_{12} Z_{13} Z_{10} & Z_{23} Z_{20} Z_{21} & Z_{30} Z_{31} Z_{32} \\
Z_{01} Z_{02} Z_{03} & Z_{12} Z_{13} Z_{10} & Z_{23} Z_{20} Z_{21} & Z_{30} Z_{31} Z_{32}
\end{array}\right]
$$

The full construction is represented by

$$
A_{3}=Q_{43} A_{0}
$$

The final matrix has a clear pattern, whose general expression is given in Section 7 . All its rows are identical, so the final vertices of $\boldsymbol{A}_{3}$ coincide, and the general label $A_{3 b}$ is used. It is again straightforward to verify that each row sums to 1 . The final points are given directly by (15) in an expression corresponding to (10): for $0 \leq b \leq n-1$,

$$
A_{3 b}=Z_{01} Z_{02} Z_{03} A_{00}+Z_{12} Z_{13} Z_{10} A_{01}+Z_{23} Z_{20} Z_{21} A_{02}+Z_{30} Z_{31} Z_{32} A_{03} .
$$

I have purely geometric proofs of the theorem for the $n=3$ and $n=4$ cases, but the methods used do not readily extend to higher values of $n$.

\section{THE GENERAL CASE}

Notation. As earlier, the original $n$-gon is $\boldsymbol{A}_{0}$, and the constructed ones are $\boldsymbol{A}_{t}$, where $t$ $(1 \leq t \leq n-1)$ signifies the construction stage. Vertices of $n$-gon $\boldsymbol{A}_{t}$ are denoted $A_{t j}$, with $0 \leq j \leq n-1$. The model polygon and its common vertex are $\boldsymbol{Z}$ and $Y$, respectively. All subscripts (except $n$ ) are taken modulo $n$. Boldface indicates polygons, vectors, and matrices. In construction stage $t$, the matrix $\boldsymbol{M}_{n t}$ is applied to polygon $\boldsymbol{A}_{t-1}$ to create polygon $\boldsymbol{A}_{t}$. In other words,

$$
\boldsymbol{A}_{t}=\boldsymbol{M}_{n t} \boldsymbol{A}_{t-1}, \quad 1 \leq t \leq n-1 .
$$

Polygon properties. No numbered vertices of $\boldsymbol{Z}$ may coincide, because of (2); $\boldsymbol{Z}$ is otherwise general. The point $Y$ can be anywhere in the plane of $\boldsymbol{Z}$ without restriction. The original $n$-gon $\boldsymbol{A}_{0}$ is completely general; the theorem is still true if two or more of its vertices coincide, producing a degenerate $n$-gon. (If $\boldsymbol{A}_{0}$ is reduced to two distinct vertices, $n$-gon $\boldsymbol{A}_{n-2}$ is similar to $\boldsymbol{Z}$; if all vertices of $\boldsymbol{A}_{0}$ coincide, $\boldsymbol{A}_{n-2}$ reduces to one point, which is "similar" to $\boldsymbol{Z}$ with zero size ratio.) For visual clarity only, $\boldsymbol{Z}$ and $\boldsymbol{A}_{0}$ can 
be thought of as convex, with vertices increasing counterclockwise, and with point $Y$ inside $\boldsymbol{Z}$.

For further clarification of this theorem, we provide detailed illustrations and a table of construction logic for the $n=5$ case. We also rewrite the construction matrices for $n=4$ in an enhanced form. This section will also explicitly give the general rules and proof from which these cases are derived.

Illustrations $(\boldsymbol{n}=\mathbf{5})$. Figures 3 and 4 show each of the four construction stages. In these, as well as in Figure 5, the model and theorem sections are independent, so there is no need to match them in orientation or scale.

Figure 3 (bottom) shows construction stages $t=1$ (left) and $t=2$ (right). Above each of these, the corresponding model appears, divided into the triangles used for that stage. Progressively shaded triangles match in the model and in the theorem construction. In the stage $t=2$ model (top right) the triangles overlap, but the intended shading is apparent.
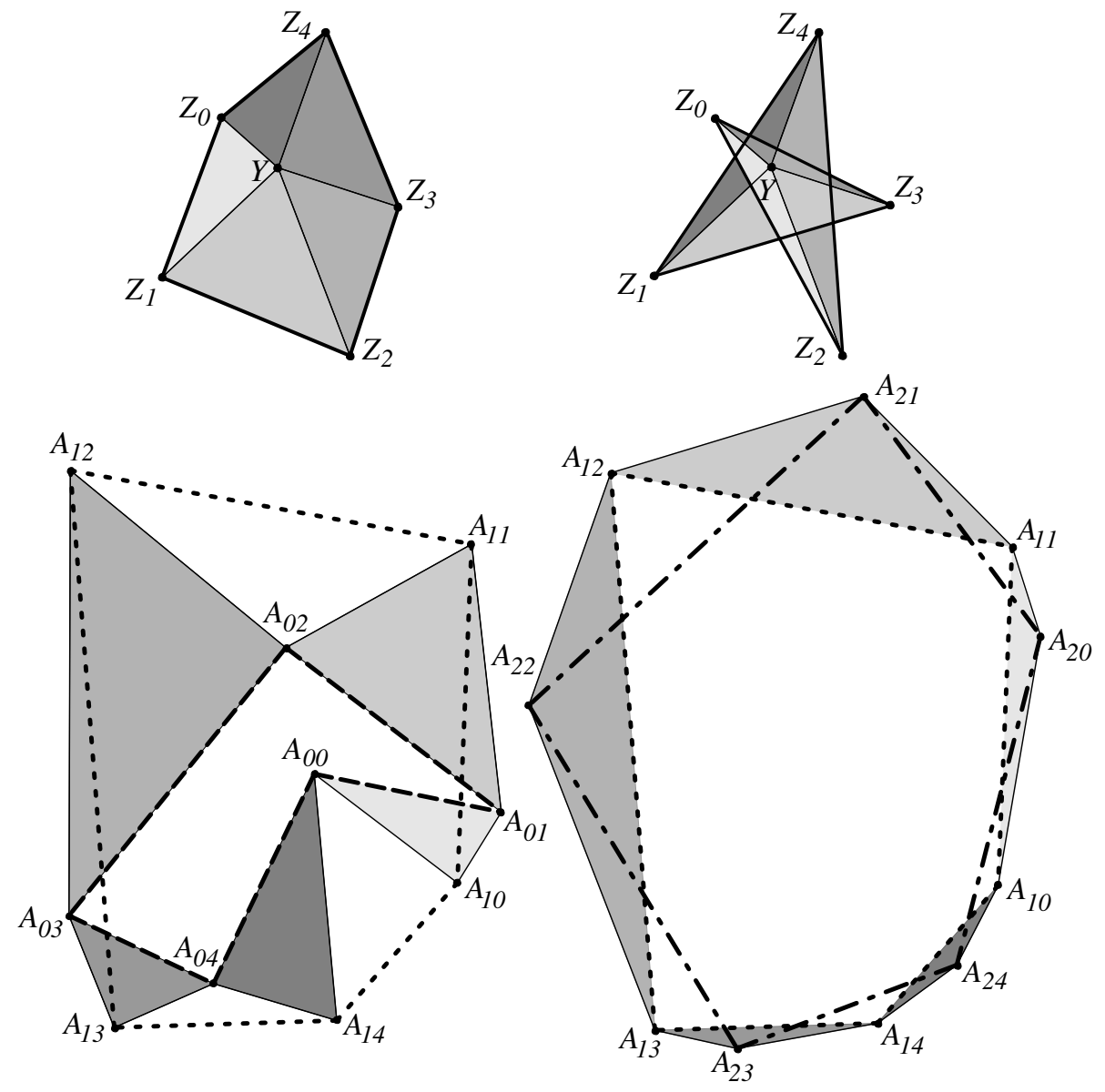

Figure 3. The case $n=5$ : model and theorem for stages $t=1$ and $t=2$.

Figure 4 (bottom) shows the stages $t=3$ and $t=4$ in the same manner. For each stage, the associated model is directly above. (Keep in mind that in the four model images, the $Z$ polygons with their the $Y$ points are congruent, differing only in the 


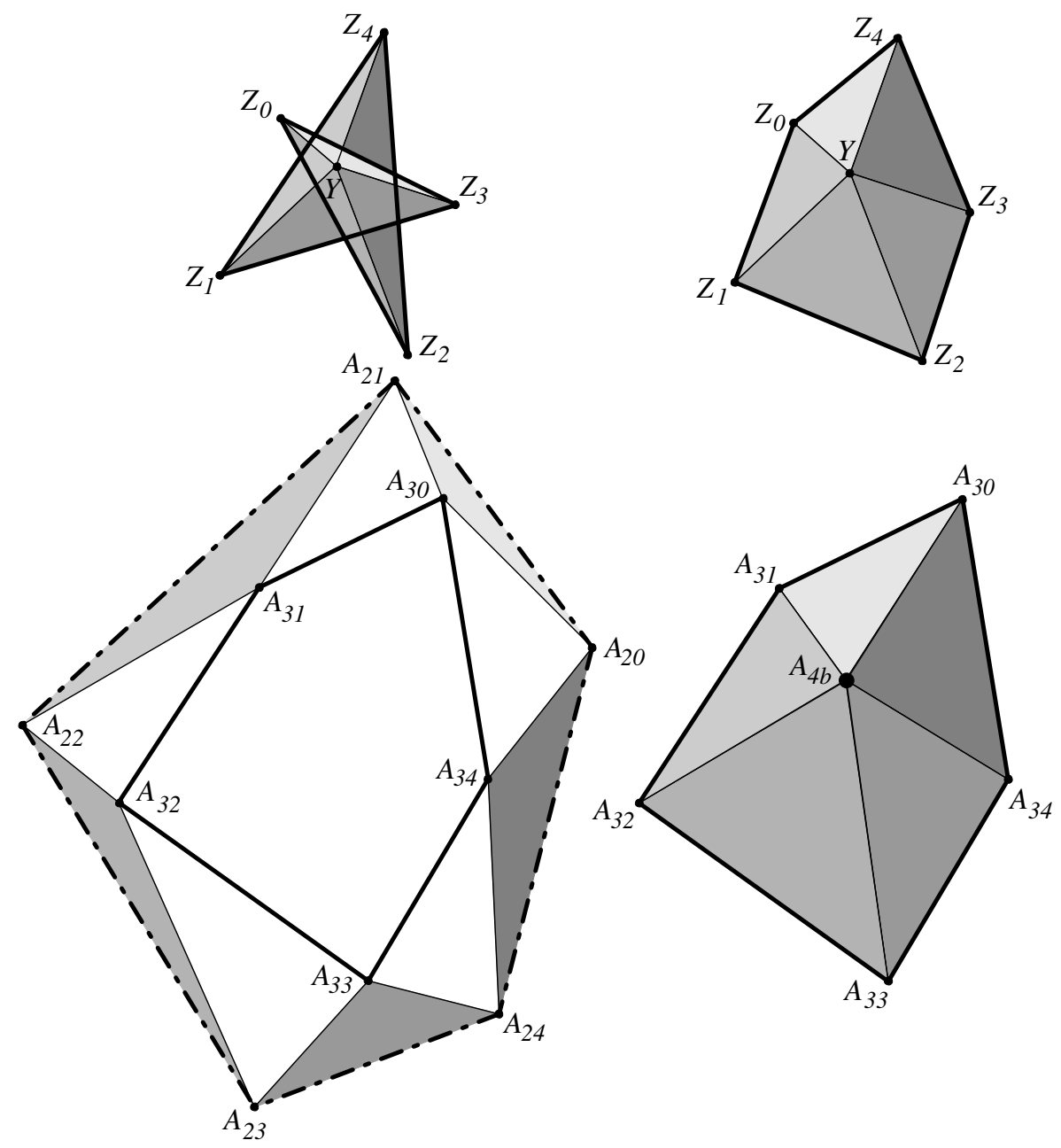

Figure 4. The case $n=5$ : model and theorem for stages $t=3$ and $t=4$.

"triangulation" of $\boldsymbol{Z}$.) In the bottom right figure, the penultimate $n$-gon $\boldsymbol{A}_{n-2}$ is similar to the model, and the final degenerate $n$-gon is represented by a point with the vertex label $A_{4 b}$.

Figure 5 illustrates the full theorem, omitting the construction triangles for clarity. The model $\boldsymbol{Z}$ and common vertex $Y$ are on the left. In the theorem figure the four $n$ gons $\boldsymbol{A}_{t}$ are shown with different line patterns. Again, $\boldsymbol{A}_{3}$ is similar to the model, and the position of $A_{4 b}$ within $\boldsymbol{A}_{3}$ corresponds to the position of $Y$ within $\boldsymbol{Z}$.

Construction table $(\boldsymbol{n}=\mathbf{5})$. Table 1 gives the construction rules for this case. A fully general statement of the rule follows an explanation of the table. Figures 3, 4, and 5 should be referred to along with these rules.

In Table 1 , the $k=0$ line in the column for $t=1$ means that a triangle similar to model triangle $Z_{0} Z_{1} Y$ is mounted on vertices $A_{01}$ and $A_{00}$ of the original polygon $\boldsymbol{A}_{0}$, with $Z_{0}$ placed on $A_{01}$ and $Z_{1}$ placed on $A_{00}$. The third vertex of the model triangle, point $Y$, corresponds to the new vertex $A_{10}$ of the next-stage polygon $\boldsymbol{A}_{1}$. Using successive lines in the stage 1 columns of Table 1, we continue counterclockwise around polygons $\boldsymbol{Z}$ and $\boldsymbol{A}_{0}$, constructing the remaining vertices of $\boldsymbol{A}_{1}$. The vertex spacing pa- 


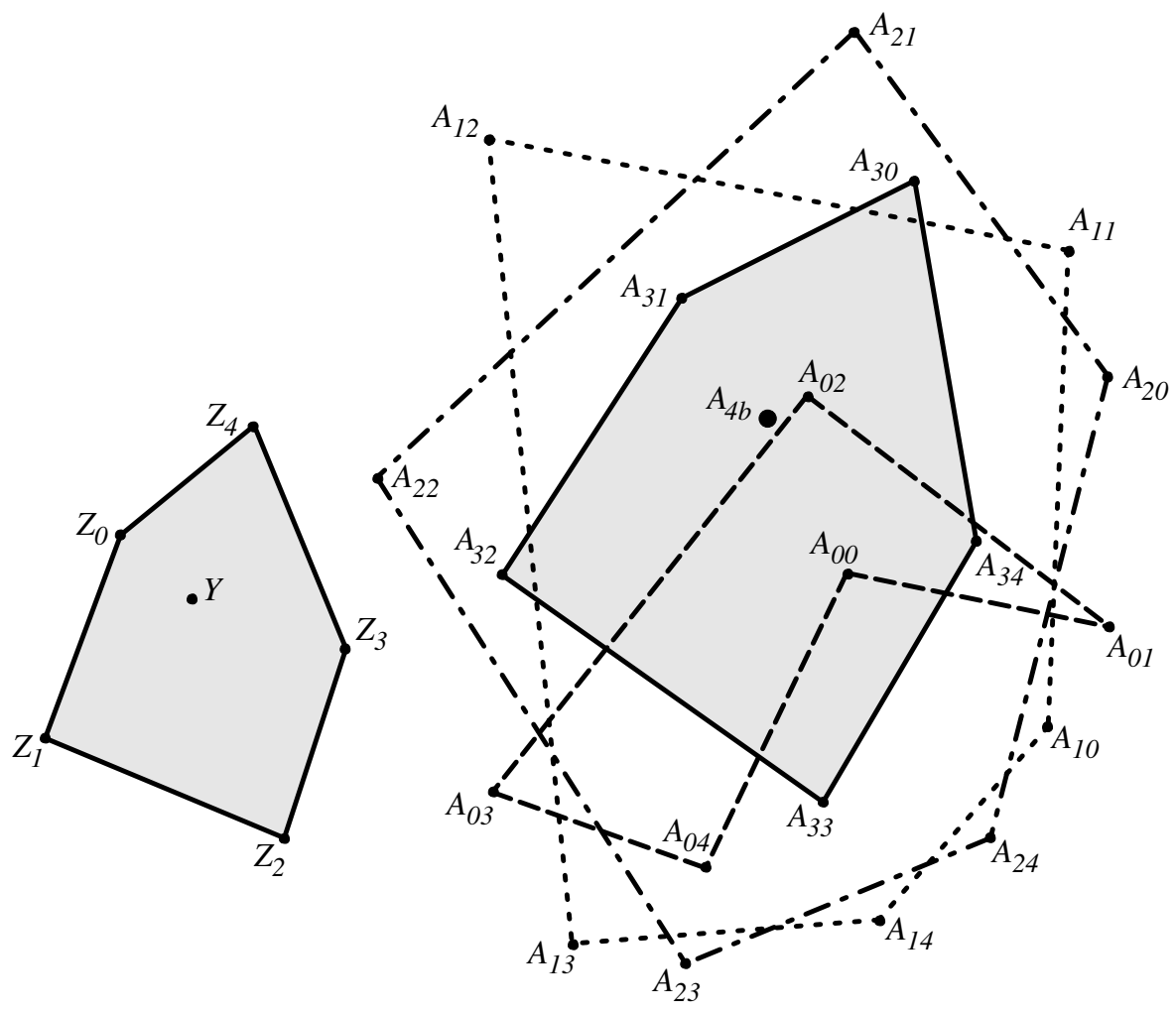

Figure 5. The case $n=5$ : model and theorem, triangles omitted.

rameter $q$ equals 1 for stage 1 , meaning that pairs of adjacent vertices of the model polygon (for example, $Z_{0}$ and $\left.Z_{1}\right)$ are used $(1-0=q)$.

Next, we build upon polygon $\boldsymbol{A}_{1}$ to make $\boldsymbol{A}_{2}$, as shown in the $t=q=2$ column. According to row $k=0$, a triangle directly similar to $Z_{0} Z_{2} Y$ is mounted on $A_{11} A_{10}$, with $Z_{0}$ going on $A_{11}$ and $Z_{2}$ going on $A_{10}$, thus defining new vertex $A_{20}$. Tracing both $\boldsymbol{Z}$ and $\boldsymbol{A}_{1}$ counterclockwise, we complete building polygon $\boldsymbol{A}_{2}$. We construct $\boldsymbol{A}_{3}$ and $\boldsymbol{A}_{4}$ in the same manner, using the rest of Table 1. At each stage, $q$ is incremented by one. For larger values of $n$, the table is extended down and to the right according to the expression for the general case, so that there are $n$ rows and $n-1$ stages.

General construction rule. Equation (16) states the general version of the second row in the table header. The main set of table entries for stage $t(1 \leq t \leq n-1)$,

TABLE 1. Construction procedure for $n=5$.

\begin{tabular}{|c|c|c|c|c|}
\hline$k$ & $\begin{array}{c}\text { Fig. } 3 \text { (left) } \\
\boldsymbol{A}_{1}=\boldsymbol{M}_{41} \boldsymbol{A}_{0} \\
\text { stage } t=q=1\end{array}$ & $\begin{array}{c}\text { Fig. } 3 \text { (right) } \\
\boldsymbol{A}_{2}=\boldsymbol{M}_{42} \boldsymbol{A}_{1} \\
\text { stage } t=q=2\end{array}$ & $\begin{array}{c}\text { Fig. } 4 \text { (left) } \\
\boldsymbol{A}_{3}=\boldsymbol{M}_{43} \boldsymbol{A}_{2} \\
\text { stage } t=q=3\end{array}$ & $\begin{array}{c}\text { Fig. } 4 \text { (right) } \\
\boldsymbol{A}_{4}=\boldsymbol{M}_{44} \boldsymbol{A}_{3} \\
\text { stage } t=q=4\end{array}$ \\
\hline 0 & $Z_{0} Z_{1} Y A_{01} A_{00} A_{10}$ & $Z_{0} Z_{2} Y A_{11} A_{10} A_{20}$ & $Z_{0} Z_{3} Y A_{21} A_{20} A_{30}$ & $Z_{0} Z_{4} Y \quad A_{31} A_{30} A_{40}$ \\
\hline 1 & $Z_{1} Z_{2} Y A_{02} A_{01} A_{11}$ & $Z_{1} Z_{3} Y A_{12} A_{11} A_{21}$ & $Z_{1} Z_{4} Y A_{22} A_{21} A_{31}$ & $Z_{1} Z_{0} Y A_{32} A_{31} A_{41}$ \\
\hline 2 & $Z_{2} Z_{3} Y A_{03} A_{02} A_{12}$ & $Z_{2} Z_{4} Y A_{13} A_{12} A_{22}$ & $Z_{2} Z_{0} Y A_{23} A_{22} A_{32}$ & $Z_{2} Z_{1} Y A_{33} A_{32} A_{42}$ \\
\hline 3 & $Z_{3} Z_{4} Y A_{04} A_{03} A_{13}$ & $Z_{3} Z_{0} Y A_{14} A_{13} A_{23}$ & $Z_{3} Z_{1} Y A_{24} A_{23} A_{33}$ & $Z_{3} Z_{2} Y \quad A_{34} A_{33} A_{43}$ \\
\hline 4 & $Z_{4} Z_{0} Y A_{00} A_{04} A_{14}$ & $Z_{4} Z_{1} Y A_{10} A_{14} A_{24}$ & $Z_{4} Z_{2} Y A_{20} A_{24} A_{34}$ & $Z_{4} Z_{3} Y A_{30} A_{34} A_{44}$ \\
\hline
\end{tabular}


skip parameter $q$, and row $k(0 \leq k \leq n-1)$, are as follows: model triangle vertices $Z_{k}, Z_{k+q}$, and $Y$ correspond to theorem triangle vertices $A_{t-1, k+1}, A_{t-1, k}$, and $A_{t k}$, respectively. In all cases of the present theorem, $q(t)=t$, and vertex subscripts are taken modulo $n$.

Theorem 1. Starting with an arbitrary plane polygon $\boldsymbol{A}_{0}$, using triangles from a model $n$-gon $\boldsymbol{Z}$, and constructing successive polygons $\boldsymbol{A}_{k}$ according to the general construction rule, polygon $\boldsymbol{A}_{n-2}$ is similar to $\boldsymbol{Z}$ and polygon $\boldsymbol{A}_{n-1}$ is a single point.

Proof. First we explicitly define the form of each of the $n-1 n$-by- $n$ construction matrices, and then prove that their product results in a form similar to (13) in which all the rows are identical. This shows that the final polygon is one point. The proof of this is linked with the distribution of zero cells in the intermediate product matrices, so a statement is made about the latter and is proved as part of the main theorem.

We consider patterns in the partial products $Q_{n t}, 0 \leq t \leq n-1$. These products were shown for $n=4$ in (12) and (13). We rewrite those here in (17), (18), and (19), using two simple conventions that simplify the analysis. First, in contrast with (2), where $Z_{u v}$ is defined only for $u \neq v$, we introduce the quantity $Z_{u u}$ to stand for 1 . Second, we display in brackets certain zero entries in $\boldsymbol{Q}_{n t}$ in order to clarify patterns in the matrices and simplify certain expressions. Using these conventions, we rewrite the product matrix $\boldsymbol{Q}_{41}$ and its successors, at the same time rearranging the terms within each entry:

$$
\begin{aligned}
\boldsymbol{Q}_{41}= & \boldsymbol{M}_{41}=\left[\begin{array}{cccc}
Z_{00} Z_{01} & Z_{10} Z_{11} & {\left[Z_{20} Z_{21}\right]} & {\left[Z_{30} Z_{31}\right]} \\
{\left[Z_{01} Z_{02}\right]} & Z_{11} Z_{12} & Z_{21} Z_{22} & {\left[Z_{31} Z_{32}\right]} \\
{\left[Z_{02} Z_{03}\right]} & {\left[Z_{12} Z_{13}\right]} & Z_{22} Z_{23} & Z_{32} Z_{33} \\
Z_{03} Z_{00} & {\left[Z_{13} Z_{10}\right]} & {\left[Z_{23} Z_{20}\right]} & Z_{33} Z_{30}
\end{array}\right], \\
\boldsymbol{Q}_{42}= & \boldsymbol{M}_{42} \boldsymbol{Q}_{41}=\boldsymbol{M}_{42} \boldsymbol{M}_{41}= \\
& {\left[\begin{array}{cccc}
Z_{00} Z_{01} Z_{02} & Z_{10} Z_{11} Z_{12} & Z_{20} Z_{21} Z_{22} & {\left[Z_{30} Z_{31} Z_{32}\right]} \\
{\left[Z_{01} Z_{02} Z_{03}\right]} & Z_{11} Z_{12} Z_{13} & Z_{21} Z_{22} Z_{23} & Z_{31} Z_{32} Z_{33} \\
Z_{02} Z_{03} Z_{00} & {\left[Z_{12} Z_{13} Z_{10}\right]} & Z_{22} Z_{23} Z_{20} & Z_{32} Z_{33} Z_{30} \\
Z_{03} Z_{00} Z_{01} & Z_{13} Z_{10} Z_{11} & {\left[Z_{23} Z_{20} Z_{21}\right]} & Z_{33} Z_{30} Z_{31}
\end{array}\right], }
\end{aligned}
$$

and

$$
\begin{aligned}
\boldsymbol{Q}_{43}= & \boldsymbol{M}_{43} \boldsymbol{Q}_{42}=\boldsymbol{M}_{43} \boldsymbol{M}_{42} \boldsymbol{M}_{41}= \\
& {\left[\begin{array}{lllll}
Z_{00} Z_{01} Z_{02} Z_{03} & Z_{10} Z_{11} Z_{12} Z_{13} & Z_{20} Z_{21} Z_{22} Z_{23} & Z_{30} Z_{31} Z_{32} Z_{33} \\
Z_{01} Z_{02} Z_{03} Z_{00} & Z_{11} Z_{12} Z_{13} Z_{10} & Z_{21} Z_{22} Z_{23} Z_{20} & Z_{31} Z_{32} Z_{33} Z_{30} \\
Z_{02} Z_{03} Z_{00} Z_{01} & Z_{12} Z_{13} Z_{10} Z_{11} & Z_{22} Z_{23} Z_{20} Z_{21} & Z_{32} Z_{33} Z_{30} Z_{31} \\
Z_{03} Z_{00} Z_{01} Z_{02} & Z_{13} Z_{10} Z_{11} Z_{12} & Z_{23} Z_{20} Z_{21} Z_{22} & Z_{33} Z_{30} Z_{31} Z_{32}
\end{array}\right] }
\end{aligned}
$$

a For $t=1$ through $n-1, Q_{n t}$ has $n-t-1$ zeroes in each row and column; this will be shown true for any $n$. Each entry in $\boldsymbol{Q}_{n t}$ is the product of $t+1$ terms, including the $Z_{u и}=1$ term. Having written out the zero entries in a form resembling the other entries (and enclosing them in brackets), we observe that a matrix entry is zero if it contains no $Z_{u u}$ term. Also observe the clear pattern of subscripts as one traverses a matrix row. Further, as one steps down in any column, the entry loses the term on the left and gains one on the right; the new term's second subscript is incremented (modulo $n$ ) with respect to the one immediately above or to the left. These patterns are evident 
only because we introduced the $Z_{u u}$ s and the zero entries in brackets; they allow us to describe with ease the general $\boldsymbol{Q}$ matrices.

Mimicking what we did in (19), we now write a general expression for entries in the $\boldsymbol{Q}$ matrices. We define

$$
\boldsymbol{Q}_{n t}=\boldsymbol{M}_{n t} \boldsymbol{M}_{n, t-1} \cdots \boldsymbol{M}_{n 1}
$$

for $0 \leq t \leq n-1$. The entire construction is represented by $\boldsymbol{Q}_{n, n-1}$, so that

$$
\boldsymbol{A}_{n-1}=\boldsymbol{Q}_{n, n-1} \boldsymbol{A}_{0},
$$

which is a general version of (8) and (14). Taking a cue from (17) through (19), we can write (with proof to follow)

$$
\left(\boldsymbol{Q}_{n t}\right)_{i j}=Z_{j i} Z_{j, i+1} \cdots Z_{j, i+t}=\prod_{s=i}^{i+t} Z_{j s} \quad[t+1 \text { terms }]
$$

for $0 \leq i, j \leq n-1$, taking subscripts modulo $n$. An integral part of the proposition (22) is that $\left(\boldsymbol{Q}_{n t}\right)_{i j}$ is defined to be zero unless the product contains a $Z_{j j}(=1)$ term. From (22) it is clear that such a term is present if and only if $j$ has one of the values (modulo $n) i, i+1, i+2, \ldots, i+t$.

It may be helpful to give a few examples of products $\boldsymbol{Q}_{n t}$ that can be checked against (17)-(19). For $n=4, t=1, i=3, j=2$, (22) gives $\left(\boldsymbol{Q}_{41}\right)_{32}=Z_{23} Z_{20}$, which contains no $Z_{j j}$ term and therefore is 0 according to the preceding definition of $\left(Q_{n t}\right)_{i j}$, agreeing with (17). For $n=4, t=3$, and any $i$ and $j$, (22) gives $\left(\boldsymbol{Q}_{43}\right)_{i j}=Z_{j i} Z_{j, i+1} Z_{j, i+2} Z_{j, i+3}$, so $\left(\boldsymbol{Q}_{43}\right)_{i j}$ contains a complete set of second subscripts modulo $n$, and therefore contains a $Z_{j j}$ term and has the same value for any $i$. In other words, all rows are equal.

Before proceeding with the proof of (22), we need a formula for entries of the construction matrices $\boldsymbol{M}_{n t}$. These are given as follows (don't forget that in $Z_{k l}$ the subscripts $k$ and $l$ are to be taken modulo $n$ ):

$$
\left(\boldsymbol{M}_{n t}\right)_{i j}= \begin{cases}Z_{i, t+j}=Z_{i, t+i} & \text { if } j=i \\ Z_{j+t-1, i}=Z_{t+i, i} & \text { if } j=i+1, \\ 0 & \text { otherwise. }\end{cases}
$$

This formula can be checked against the entries in (11).

To begin the proof by induction, we first show that (22) holds for $t=1$ : in this case (22) asserts that $\left(\boldsymbol{Q}_{n 1}\right)_{i j}=\left(\boldsymbol{M}_{n 1}\right)_{i j}=Z_{j i} Z_{j, i+1}$ (two terms), where the first equality comes from (20) and the second from (22). This must agree with the basic definition of $\left(\boldsymbol{M}_{n 1}\right)_{i j}$ from (23). For $j=i$, (22) gives $\left(\boldsymbol{Q}_{n 1}\right)_{i i}=Z_{i i} Z_{i, i+1}$, agreeing with (23) since $Z_{i i}=1$. For $j=i+1$, (22) is $\left(\boldsymbol{Q}_{n 1}\right)_{i, i+1}=Z_{i+1, i} Z_{i+1, i+1}=Z_{i+1, i}$, again agreeing with (23).

Next, we do the main inductive step in the proof, showing that (22) still holds when $t$ increments to $t+1$. According to (20),

$$
\boldsymbol{Q}_{n, t+1}=\boldsymbol{M}_{n, t+1} \boldsymbol{Q}_{n t} .
$$

Using the standard matrix multiplication formula, (24) gives

$$
\left(\boldsymbol{Q}_{n, t+1}\right)_{i j}=\sum_{k=0}^{n-1}\left(\boldsymbol{M}_{n, t+1}\right)_{i k}\left(\boldsymbol{Q}_{n t}\right)_{k j} .
$$


Each row of matrix $\boldsymbol{M}_{n l}$ has only two nonzero terms $\left(\boldsymbol{M}_{n l}\right)_{i k}$, those with $k=i$ and $k=i+1$, so (25) becomes

$$
\left(\boldsymbol{Q}_{n, t+1}\right)_{i j}=\left(\boldsymbol{M}_{n, t+1}\right)_{i i}\left(\boldsymbol{Q}_{n t}\right)_{i j}+\left(\boldsymbol{M}_{n, t+1}\right)_{i, i+1}\left(\boldsymbol{Q}_{n t}\right)_{i+1, j}
$$

Using (23) for the terms of matrices $\boldsymbol{M}_{n l}$ and (22) for the $\boldsymbol{Q}_{n m}$, we have

$$
\left(\boldsymbol{Q}_{n, t+1}\right)_{i j}=Z_{i, i+t+1}\left(Z_{j i} Z_{j, i+1} \cdots Z_{j, i+t}\right)+Z_{i+t+1, i}\left(Z_{j, i+1} Z_{j, i+2} \cdots Z_{j, i+t+1}\right),
$$

where each expression in parentheses has $t+1$ terms. Gathering terms, we have

$$
\left(\boldsymbol{Q}_{n, t+1}\right)_{i j}=\left(Z_{j, i+1} \cdots Z_{j, i+t}\right)\left(Z_{i, i+t+1} Z_{j, i}+Z_{i+t+1, i} Z_{j, i+t+1}\right) .
$$

Using (4) with $u=i, v=i+t+1$, and $w=j$ simplifies the second set of terms, reducing (26) to

$$
\left(\boldsymbol{Q}_{n, t+1}\right)_{i j}=Z_{j i} Z_{j, i+1} \cdots Z_{j, i+t} Z_{j, i+t+1}
$$

for $0 \leq i, j \leq n-1$, with $t+2$ terms including a term of the type $Z_{j j}$ if there is one. This is the same as (22), with $t+1$ replacing $t$, so the formula for $\boldsymbol{Q}_{n t}$ extends from $t$ to $t+1$. Then by induction it holds for all $t$, and since $n$ was not assigned a specific value, it also holds for all $n$.

When $t=n-2$, (27) becomes

$$
\left(\boldsymbol{Q}_{n, n-1}\right)_{i j}=Z_{j i} Z_{j, i+1} \cdots Z_{j, i+n-1} \quad[n \text { terms }] .
$$

All values of the second subscript (modulo $n$ ) are present in (28), ensuring that the product is not a function of the row index $i$. (Changing $i$ just permutes the terms.) Therefore all rows are equal.

Finally, (27) shows that when $t$ increases by one, the range of $j$ for which a $Z_{j j}$ turns up in (22) also increases by one. As a result, there is one more nonzero entry in each row and column of $\boldsymbol{Q}_{n, t+1}$ than occurs in $\boldsymbol{Q}_{n t}$, as is required for the consistency of (22). This completes the inductive part of the proof.

Thus far we have proved that all rows of the final product matrix $\boldsymbol{Q}_{n, n-1}$ are equal, so the last construction stage results in a single point. The only remaining task is to prove that the penultimate stage delivers a polygon similar to the model. To do this, we express the final degenerate polygon $\boldsymbol{A}_{n-1}$ in terms of the preceding one, $\boldsymbol{A}_{n-2}$. The basic matrix relation is (16), in which we set $t=n-1$. The entries in $\boldsymbol{M}_{n, n-1}$ come from (23). We also need the definitions of the $Z_{u v}$ given in (2). The two nonzero terms of $\boldsymbol{M}_{n, n-1} \boldsymbol{A}_{n-2}$ are then

$$
A_{n-1, i}=\frac{\left(Z_{i}-Y\right) A_{n-2, i}-\left(Z_{n-1+i}-Y\right) A_{n-2, i+1}}{Z_{i}-Z_{n-1+i}} .
$$

This equation represents one of the triangle constructions used in the last stage. To establish similarity between the model $\boldsymbol{Z}$ and the polygon $\boldsymbol{A}_{n-2}$, we apply the same construction to part of $\boldsymbol{Z}$ itself, substituting $Z_{n-1+i}$ for $A_{n-2, i}$ and $Z_{i}$ for $A_{n-2, i+1}$. Putting these $Z$ vertices into (29) and simplifying gives

$$
\frac{\left(Z_{i}-Y\right) Z_{n-1+i}-\left(Z_{n-1+i}-Y\right) Z_{i}}{Z_{i}-Z_{n-1+i}}=Y .
$$


Equations (29) and (30) demonstrate that the same linear construction that takes vertices $A_{n-2, i}$ and $A_{n-2, i+1}$ into $A_{n-1, i}$, also takes vertices $Z_{n-1+i}$ and $Z_{i}$ into $Y$. Therefore the triangles $A_{n-2, i} A_{n-2, i+1} A_{n-1, i}$ and $Z_{n-1+i} Z_{i} Y$ are similar. Since the subscript $i$ is general, any given triangle in $\boldsymbol{A}_{n-2}$ is similar to a corresponding one in $\boldsymbol{Z}$. Since $Y$ is common to all model triangles, and since we already showed that all constructions on $\boldsymbol{A}_{n-2}$ lead to the common point $A_{n-1, b}$, it follows that $\boldsymbol{A}_{n-2}$ is similar to $\boldsymbol{Z}$. For an example, let $n=5$ and $i=3$. Then the similarity applies to triangles $A_{33} A_{34} A_{4 b}$ (with $b=3$ ) and $Z_{2} Z_{3} Y$. Figure 4 (right) confirms this. The proof is now complete.

Although not part of the theorem itself, we can write an explicit formula for the ultimate common point in terms of the coefficients $Z_{j k}$ and the initial polygon $\boldsymbol{A}_{0}$. We adapt the general matrix multiplication formula to (21), where the vertex vectors consist only of column 0 and the second subscript refers to the row entry of the vector. Then

$$
A_{n-1, b}=\sum_{k=0}^{n-1}\left(\boldsymbol{Q}_{n, n-1}\right)_{b k} A_{0 k} .
$$

For the $Q$ terms we use the right part of (22) with $t=n-1$ :

$$
\left(\boldsymbol{Q}_{n, n-1}\right)_{b k}=\prod_{s=b}^{b+n-1} Z_{k s}=\prod_{s=0}^{n-1} Z_{k s}
$$

The second equality obtains because in the first product, the $s$ subscript ranges through all $n$ values and subscripts are taken modulo $n$. It follows that any integer can be added to both limits of the second product without changing the result. (Changing both limits by the same amount just permutes the terms.) Combining (31) and (32), we have

$$
A_{n-1, b}=\sum_{k=0}^{n-1}\left(A_{0 k} \prod_{s=0}^{n-1} Z_{k s}\right) .
$$

Every product in this sum will contain a $Z_{k k}=1$ term. With this in mind, we remark that (33) is a generalization of (10) and (15), which are written without the $Z_{k k}=1$ term. From (33) and definition (2) we see that the final point $A_{n-1, b}$ is linear in the vertices of the original $n$-gon $\boldsymbol{A}_{0}$ but highly nonlinear in all parts of the model.

8. THE PDN-THEOREM. We now verify that the new theorem reduces to the PDNtheorem in the situation where the model is regular. The main theorem shows that the final $n$-gon is similar to the model, which in this case makes it regular, regardless of the location of $Y$. In the discussion of equations (29) and (30), we proved that each constituent triangle of $\boldsymbol{A}_{n-2}$ is similar to a corresponding one of $\boldsymbol{Z}$. This immediately implies that, if $Y$ is at the centroid of $\boldsymbol{Z}$, then the final point $A_{n-1, b}$ is at the centroid of $\boldsymbol{A}_{n-2}$.

More generally, we now show that in the case of the PDN-theorem, the centroid is invariant with respect to any stage of construction. Since the scale and angular offset of the model are not important, we can let $Y=0$ and express the model's $j$ th vertex $(0 \leq j \leq n-1)$ in the form

$$
Z_{j}=e^{2 j \pi i / n}
$$


In tandem with (2) this gives

$$
Z_{j k}=1 /\left(1-e^{2(j-k) \pi i / n}\right) .
$$

Now define $S_{t}$ to be the sum of the vertices of $n$-gon $t$. We wish to find $S_{t}$ in terms of $S_{t-1}$. First, writing (16) as a matrix-vector product much like (31), we get

$$
A_{t k}=\sum_{j=0}^{n-1}\left(M_{n t}\right)_{k j} A_{t-1, j} .
$$

Then

$$
S_{t}=\sum_{k=0}^{n-1} \sum_{j=0}^{n-1}\left(M_{n t}\right)_{k j} A_{t-1, j}=\sum_{j=0}^{n-1} A_{t-1, j} \sum_{k=0}^{n-1}\left(M_{n t}\right)_{k j},
$$

where the order of summation has been reversed in the right-hand expression. Note that the second sum is over one column of $M_{n t}$, which like any row has only two nonzero terms. For a fixed $j$ these terms are $i=j$ and $i=j-1$, modulo $n$. The nonzero terms in (23) are then $\left(M_{n t}\right)_{j j}=Z_{j, t+j}$ and $\left(M_{n t}\right)_{j-1, j}=Z_{t+j-1, j-1}$. Now we use the $Z$ values for the regular model from (34) to evaluate the second sum in (35):

$$
\sum_{k=0}^{n-1}\left(M_{n t}\right)_{k j}=\frac{1}{\left(1-e^{2 t \pi i / n}\right)}+\frac{1}{\left(1-e^{-2 t \pi i / n}\right)}=1 .
$$

Then

$$
S_{t}=\sum_{j=0}^{n-1}\left(A_{t-1}\right)_{j}=S_{t-1},
$$

so that the vertex sum of stage $t$ equals that of stage $t-1$. Therefore centroids are invariant from stage to stage.

This discussion has not covered commutation of the construction stages, another important property of the PDN-theorem. Commutation can be proved straightforwardly by taking the product of matrices representing two different stages of construction. Call them $\boldsymbol{M}_{n t}$ and $\boldsymbol{M}_{n u}$. We compute $\boldsymbol{P}_{t u}=\boldsymbol{M}_{n t} \boldsymbol{M}_{n u}$ and $\boldsymbol{P}_{u t}=\boldsymbol{M}_{n u} \boldsymbol{M}_{n t}$ using the matrix definition of (23) and the standard multiplication formula. Substituting the special values for $Z_{j k}$ according to (34), we find that $\boldsymbol{P}_{t u}=\boldsymbol{P}_{u t}$. Therefore any two stages commute. Now any order of construction can be obtained by starting with the specific product order of (20) with $t=n-1$, and swapping adjacent terms until the desired permutation is reached. The worst-case number of swaps, which would be required to reverse the order of all $n-1$ construction steps, is $(n-1)(n-2) / 2$.

9. CONCLUDING REMARKS. In the preceding development, actual properties of complex quantities are needed only in the PDN case, where it is convenient to define regular model polygons in terms of exponentials. In the general case we needed only to know that lengths and angles can be represented by complex numbers, as in (1), and that those complex numbers can be manipulated by algebraic means. Also, the only matrix algebra tools we needed were multiplication, and some care in manipulating subscripts. This makes the proof relatively elementary, although not particularly simple. 
A worthwhile goal would be a synthetic proof of the new theorem, despite the presence of the variable $n$. So far as I know there are no truly synthetic proofs of theorems involving general $n$-gons. There are a few that come somewhat close, where induction on $n$ can be used [15]. It appears that inductive methods are not useful here. Synthetic proofs are sometimes better at affording insights and showing directions for generalization, compared with proofs using complex numbers and matrices.

Having conjectured the present theorem, I performed visual checks on it using Geometer's Sketchpad for $n \leq 7$. It certainly seemed to be true, but a synthetic proof for $n>4$ appeared infeasible. I then went to matrix methods, although I regard them as foreign to the spirit of geometry (if frequently indispensable). Seeing a pattern in the matrix products, I verified them using Mathematica for $n \leq 7$ before constructing the general proof. Using modern mathematical tools made the development much easier, especially in the conjecture stage.

At the other end of the spectrum, as $n$ takes on large values, polygons $\boldsymbol{A}_{0}$ and $\boldsymbol{Z}$ can both be made to approach closed, smooth curves. The theorem is meaningful for any finite value of $n$. It provides a construction using triangles that takes the arbitrary curve $\boldsymbol{A}_{0}$ into $\boldsymbol{A}_{n-2}$, a curve that is "similar" to $\boldsymbol{Z}$ but not related in shape to $\boldsymbol{A}_{0}$. Related questions involving smooth curves can easily be posed. One challenge would be to apply continuous-variable analysis to define and prove the limiting case. Another is to see under what conditions, if any, the intermediate polygons remain bounded as $n \rightarrow \infty$.

Another goal would be to establish a three-dimensional counterpart to the new theorem. Obvious questions are what plane(s) to place the triangles in and what an outward or inward construction should mean (perhaps away from or toward the polygon's centroid). There are a few theorems applying to polygons in $\mathbb{R}^{3}[\mathbf{1 0}]$, but there is no assurance that one involving triangle constructions on $n$-gons is possible (or impossible).

A relatively short-term task, now under way, is to relax conditions on the construction, increasing the new theorem's generality still more. In addition, I have found variations of the present theorem in which the vertex spacings follow different rules.

\section{REFERENCES}

1. H. F. Baker, A remark on polygons, J. London Math. Soc. 17 (1942) 162-164.

2. R. Barbara, A corollary of Napoleon's theorem, Math. Gazette (July, 1998) 297-298.

3. A. Bogomolny, Napoleon's theorem, http://www.cut-the-knot.com/ctk/Napolegon.shtml.

4. G. Chang, A proof of Douglas and Neumann by circulant matrices, Houston J. Math. 8 (1982) 15-18.

5. G. Chang and P. Davis, A circulant formulation of the Napoleon-Douglas-Neumann theorem, Linear Alg. Appl. (1983) 87-95.

6. P. J. Davis, Circulant Matrices, Chelsea Publishing, New York, 1994.

7. M. De Villiers, Generalizing Van Aubel using duality, Math. Mag. 73 (2000) 303-307. Web: http://mzone.mweb.co.za/residents/profmd/aubel.pdf

8. J. Douglas, Geometry of polygons in the complex plane, J. Math. and Phys. 19 (1940) 93-130.

9. $\quad$ On linear polygon transformations, Bull. Amer. Math. Soc. 46 (1940) 551-560.

10. - A theorem on skew pentagons, Scripta Math. 25 (1960) 5-9.

11. R. L. Finney, Dynamic proofs of Euclidean theorems, Math. Mag. 43 (1970) 177-185.

12. J. C. Fisher, D. Ruoff, and J. Shiletto, Polygons and polynomials, in The Geometric Vein, P. Davis, A. Grunbaum, and F. Sherk, eds., Springer-Verlag, New York, 1980, 321-333.

13. M. Fox, Napoleon triangles and adventitious angles, Math. Gazette (November, 1998) 413-422.

14. J. Garfunkel, problem PME 553, Pi Mu Epsilon J. 7 (1983) 614.

15. L. I. Golovina and I.M. Yaglom, Induction in Geometry, D.C. Heath \& Co., Boston, 1963.

16. P. J. Kelly and D. Meriell, Concentric polygons, this MonThLY 71 (1964) 37-41.

17. R. I. Loebl, The non-commutative triangle inequality fails, this MonTHLY, 83 (1976) 259-260.

18. D. Merriell, Further remarks on concentric polygons, this MONTHLY 72 (1965) 960-965.

19. B. H. Neumann, Some remarks on polygons, J. London Math. Soc. 16 (1941) 551-560. 
20. - A remark on polygons, J. London Math. Soc. 17 (1942) 165-166.

21. P. Pech, The harmonic analysis of polygons and Napoleon's theorem, J. Geometry and Graphics $\mathbf{5}$ (2001) 13-22.

22. D. Pedoe, Geometry, A Comprehensive Course, Dover, New York, 1988.

23. K. Petr, Ein Satz über Vielecke, Arch. Math. Physik 13 (1908) 29-31.

24. J. F. Rigby, Napoleon revisited, J. Geom. 33 (1988) 129-146.

25. E. T. Wong, Polygons, circulant matrices, and Moore-Penrose Inverses, this MonTHLY 88 (1981) 509515.

26. Y. C. Wong, Some extensions of the Douglas-Neumann theorem for concentric polygons, this MonTHLY 75 (1968) 470-482.

27. J. E. Wetzel, Converses of Napoleon's theorem, this Monthly 99 (1992) 339-351.

28. I. M. Yaglom, Geometric Transformations, Random House, New York, 1962.

29. Geometric Transformations II, Mathematical Association of America, Washington, D.C., 1968.

STEPHEN B. GRAY earned a B.S. at the University of Pennsylvania and did graduate work at Harvard University in physics and mathematics. His career has been in designing advanced hardware and software in the field of computer graphics. More recently, after building a house in Santa Monica, California, he has returned to his favorite subject, geometry.

stevebg@adelphia.net 